Isidore Fleischer, Universite de Montreal, Centre de recherches mathematiiques (CRM), C.P. 6128, succursale Centre-Ville, Monteal (Quebec) H3C 3J7, Canada. e-mail: fleischi@crm.umontreal.ca John E. Porter, Department of Mathematics, Auburn University, Auburn, AL 36849-5310 e-mail: porteje@auburn.edu

\title{
CONVERGENCE OF METRIC SPACE-VALUED BV FUNCTIONS
}

\begin{abstract}
Chistyakov has proved "Helly's selection theorem" - a uniformly BV sequence has a pointwise convergent subsequence - for Banach-(resp. continuous, group-) valued functions from a real interval into a compact subset. We extend, dispensing with continuity, to arbitrary real subsets and lighten compactness of the range to pointwise precompactness (which answers one of his questions). In addition, we accomplish his selection more generally for complete metric-set-valued BV maps with closed graphs which are pointwise compact on dense subsets of their domains.
\end{abstract}

\section{Introduction}

Variation may be defined for a metric space-valued function $f$ of a real variable just as for real-valued ones as

$$
V_{E}(f)=\sup \sum_{i=1}^{m} d\left(f\left(t_{i}\right), f\left(t_{i-1}\right)\right)
$$

over all finite $t_{0} \leq \ldots \leq t_{i} \leq \ldots \leq t_{m}$ in $E \subset R$ (d is the metric). Where finite, this may be shown to have the same real-valued properties: non-negative, additive on contiguous intervals (hence non-decreasing), continuous for continuous functions, change of variables: $V_{E}(g \circ \phi)=V_{\phi(E)}(g)$ for nondecreasing $\phi$, and "lower semi-continuity": $V_{E} \lim \left(f_{n}\right) \leq \liminf V_{E}\left(f_{n}\right) \leq \sup V_{E}\left(f_{n}\right)$.

Key Words: bounded variation,pointwise precompact

Mathematical Reviews subject classification: Primary 26A45; Secondary 54C65, 54C60

Received by the editors September 29, 2000 
Real-valued functions of bounded variation admit a "Jordan decomposition" as the sum of a non-decreasing and a non-increasing part which vary (i.e. which are non-constant) on disjoint subsets of the domain. The bounded variation function could alternatively be obtained by applying to its (total) variation, which is the difference of these, the function identity on the increments where the non-decreasing part varies and negative identity on the increments in the complement. This function is (in Chistyakov's terminology) natural (a "curve-isometry" in $[\mathrm{M}]$ ) in that its variation over every interval is equal to that interval's length. Such a function is in particular Lip(1); conversely the composite of every $\operatorname{Lip}(1)$ with a nondecreasing function has bounded variation.

Our argument is based on the following Federer-Moreau-Chistyakov structure theorem:

Theorem 1.1. A function $f: E \rightarrow X$ has bounded variation if and only if it factors as $g \circ \phi$ where $\phi: E \rightarrow R$ is its total variation and $g: \phi(E) \rightarrow X$ is natural (or even Lipschitz).

For the proof, observe that the variation factors $f$; the quotient $g: \phi(E) \rightarrow$ $X$ is natural by the change of variables formula. Proved in $[\mathrm{F}, \mathrm{p} .110]$ with g Lip(1), in [M, Prop. 15.1] for Banach space-valued functions and in [Ch, Theorem. 3.1] for metric space-valued functions.

The authors would like to thank Jack Brown in whose seminar these results were developed and Strashimir Popvassilev for his excellent notes.

\section{Helly's Theorem}

Lemma 2.1. A uniformly bounded sequence of non-decreasing real-valued functions has a pointwise convergent sub-sequence.

Proof. The proof is identical to [N pp. 221-222] starting with any countable dense subset.

Lemma 2.2. Let $\left\{\phi_{n}(t)\right\}$ be a sequence of real-valued functions such that $\phi_{n}(t) \rightarrow \phi(t)$ on $E$. Let $\left\{g_{n}(t)\right\}$ be a sequence of Lip(1) functions from the reals into a metric space $X$. Then $g_{n} \circ \phi_{n}$ converges pointwise on $E$ if and only if $g_{n}$ converges pointwise on $\phi(E)$.

Proof. Observe that $d\left(g_{n}\left(\phi_{n}(t)\right), g_{n}(\phi(t))\right) \leq\left|\phi_{n}(t)-\phi(t)\right| \rightarrow 0$ as $n \rightarrow \infty$.

Theorem 2.3. Let $\mathcal{F}$ be a sequence of functions of uniform bounded variation from $E$ to a metric space $X$ (that is, $\sup _{f \in \mathcal{F}} V_{E}(f)<\infty$ ) such that $\mathcal{F}$ is pointwise precompact (i.e., $\{f(t): f \in \mathcal{F}\}$ is precompact for every $t \in E$ ). 
Then there exists a sub-sequence $\left\{f_{n}\right\} \subset \mathcal{F}$, pointwise convergent on $E$ to a function $f: E \rightarrow X$, hence of bounded variation with $V_{E}(f) \leq \sup _{f_{1} \in \mathcal{F}} V_{E}\left(f_{1}\right)$.

Proof. Without loss of generality, we can assume $\mathrm{X}$ is a Banach space since every metric space can be embedded isometrically in a Banach space. Represent each $f \in \mathcal{F}$ as a composite $f=g \circ \phi$ where $\phi$ is the variation of $f$ and $g: \phi(E) \rightarrow X$ is a Lipschitz map with constant 1 . Since the $\phi$ 's are a uniformly bounded sequence of non-decreasing real-valued functions, they have a subsequence $\left\{\phi_{n}\right\}$ which converges pointwise to a non-decreasing function $\phi(x)$. We may assume that the corresponding $g_{n}$ 's are defined on all the reals since Lip(1) functions into Banach spaces extend as such from subsets of the reals (see [Ch Theorem 5.1, step 3]). Since $\left\{f_{n}=g_{n} \circ \phi_{n}\right\}$ is pointwise precompact on $E,\left\{g_{n}\right\}$ is pointwise precompact on $\phi(E)$ by lemma 2.2, so by the ArzelaAscoli theorem (see [D]), there exists a sub-sequence $g_{n_{k}}$ which converges on $\phi(E)$, and again by lemma $2.2 f_{n_{k}}=g_{n_{k}} \circ \phi_{n_{k}}$ converges pointwise on $E$.

\section{Set-Valued Maps}

Let $A, B \subset X$ be two nonempty closed bounded subsets of a metric space (X,d). The Hausdorff distance between $A$ and $B$ is defined by

$$
d_{H}(A, B)=\max \{e(A, B), e(B, A)\},
$$

where

$$
e(A, B)=\sup _{x \in A} \operatorname{dist}(x, B)=\sup _{x \in A} \inf _{y \in B} d(x, y) \in[0, \infty)
$$

This defines a metric on the set $\mathcal{C}$ of all nonempty closed bounded subsets of $X$.

A set-valued map from $E \subset R$ to a metric space $X$ is (for us) a map $F: E \rightarrow \mathcal{C}$. Such an $F$ has bounded variation on $E \subset R$ if

$$
V_{E}(F)=\sup \sum_{i=1}^{m} d_{H}\left(F\left(t_{i}\right), F\left(t_{i-1}\right)\right)<\infty
$$

over all finite $t_{0} \leq \ldots \leq t_{i} \leq \ldots \leq t_{m}$ in $E$; is compact-valued on a dense set if $F(t)$ is a compact subset of $X$ for every $t$ in some dense subset of $E$; has closed (compact) graph if its graph $\{(t, x) \in E \times X: x \in F(t)\}$ is closed (compact) in the product space $E \times X$. The function $f: E \rightarrow X$ is said to be a selection of the set-valued map $F: E \rightarrow \mathcal{C}$ if $f(t) \in F(t)$ for all $t \in E$.

Lemma 3.1. Let $\left\{f_{n}(x)\right\}$ be a sequence of metric space-valued functions defined "finally" (i.e. at each point all but finitely many are defined) on E. If 
the sequence is pointwise precompact on a countable subset $D \subset E$, then there is a sub-sequence which is defined finally and converges at every point of $D$.

Proof. Diagonalize the sub-sequences converging on increasing finite subsets of $D$.

Lemma 3.2. A bounded variation function to a complete metric space extends to the closure of its domain via one-sided limits (hence without increase of its variation).

Proof. This holds for both the monotone non-decreasing and the natural factors (see [F, 2.5.16, p. 109]).

Chistyakov [Ch] obtains the following conclusion only for a map with compact graph from an interval to a Banach space.

Theorem 3.3. A BV set-valued map with closed graph to a complete metric space $X$, pointwise compact on a dense subset $D$ of $E$, has a selection $f$ which goes through a given pair in the graph and whose variation is at most that of F.

Proof. Let $D=\cup D_{n}$ where each $D_{n}$ is finite and $D_{n} \subset D_{n+1}$. Define a function $f_{n}$ on $D_{n}=\left\{r_{1}<\ldots<r_{m}\right\}$ for given $f_{n}\left(r_{1}\right) \in F\left(r_{1}\right)$ by $f_{n}\left(r_{k+1}\right) \in$ $F\left(r_{k+1}\right)$ such that

$$
d\left(f_{n}\left(r_{k}\right), f_{n}\left(r_{k+1}\right)\right)=d\left(f_{n}\left(r_{k}\right), F\left(r_{k+1}\right)\right) \leq d_{H}\left(F\left(r_{k}\right), F\left(r_{k+1}\right)\right)
$$

(Remember that $F\left(r_{k+1}\right)$ is compact.) Note that the variations of $\left\{f_{n}\right\}$ are bounded by the variation of $F$. By lemma 3.1, there is a subsequence which converges pointwise to a function $f$; hence also $V_{D}(f) \leq V_{E}(F)$. By lemma 3.2 extend $f$ to a bounded variation function with $V_{E}(f) \leq V_{E}(F)$. Since the graph of $F$ is closed $f(t) \in F(t){ }^{1}$

\footnotetext{
${ }^{1}$ Added 2001: The above was submitted for publication in May 2000. In the interim, a paper by S. A. Belov and V. V. Chistyakov appeared in J. Math. Anal. Appl. vol. 249, pp. 351-366 which also extends Chistyakov's (there called) Theorem B by replacing compactness of the range with pointwise precompactness - but not as far as the above Theorem 2.3, since it is restricted to intervals whereas ours is valid for arbitrary subsets. The respective selection theorems for set-valued maps appear to be incomparable: Our Theorem 3.3 is not contained in their Theorem 2 as can be verified by the map into an infinite discrete metric space (all distances apart 1) which is a constant singleton except at one real at which it is the whole space. On the other hand, by using our Theorem 2.3 it is possible to extend their set-valued theorem from intervals to arbitrary subsets (like ours). We have determined that their proof of Theorem 1, the Helly selection principle is wrong. The error occurs in the last paragraph on p.359 where they unjustifiedly assume that the countable set of discontinuities of all the variation functions is closed.
} 


\section{References}

[1] V. V. Chistyakov, On the theory of set-valued maps of bounded variation of one real variable, Matematicheskii Sbornik 189 (1998), No. 5, 153-176.

[2] J. Dugundji, Topology, Allyn and Bacon, Inc., Boston, 1966.

[3] H. Federer, Geometric Measure Theory, Springer-Verlag, New York, 1969.

[4] J. J. Moreau, Bounded Variation in Time, in: J. J. Moreau, P. D. Panagiotopoulos, and G. Strang, eds., (Topics in Nonsmooth Mechanics, Birkhauser Verlag, 1988).

[5] I. Natanson, Theory of Functions of a Real Variable, vol. I, New York (1974). 
